\title{
Strang splitting method for Burgers-Huxley equation
}

\author{
Y. Çiçek ${ }^{\mathrm{a}, *}, \mathrm{G}$. Tanoğlu ${ }^{\mathrm{b}}$ \\ a Izmir Institute of Technology, The Graduate School of Engineering E' Sciences, Department of Mathematics, 35430 Urla, Izmir, Turkey \\ ${ }^{\mathrm{b}}$ Izmir Institute of Technology, Department of Mathematics, 35430 Urla, Izmir, Turkey
}

\section{A R T I C L E I N F O}

\section{Keywords:}

Operator splitting method

Burgers-Huxley equation

Regularity

Sobolev spaces

Error analysis

\begin{abstract}
A B S T R A C T
We derive an analytical approach to the Strang splitting method for the BurgersHuxley equation (BHE) $u_{t}+\alpha u u_{x}-\epsilon u_{x x}=\beta(1-u)(u-\gamma) u$. We proved that Srtang splitting method has a second order convergence in $H^{s}(\mathbb{R})$, where $H^{s}(\mathbb{R})$ is the Sobolev space and $s$ is an arbitrary nonnegative integer. We numerically solve the BHE by Strang splitting method and compare the results with the reference solution.
\end{abstract}

(c) 2015 Elsevier Inc. All rights reserved.

\section{Introduction}

Nonlinear partial differential equations are important in most fields of science. Burgers-Huxley equation has a great importance of being a nonlinear partial differential equation. It describes the interactions between reaction mechanisms, convection effects and diffusion transports [1]. This equation was firstly introduced by Bateman [2], then treated by Burgers [3] in a mathematical modeling of turbulence. These NPDEs are high importance in nonlinear physics. BHE is a perturbation problem by having the perturbation parameter $\epsilon \in(0,1)$. To solve the BHE various numerical techniques were applied by the researchers. In [12], they have applied a quasilinearization process which is long and complicated. Also, Zhou and Cheng [13] have applied the operator splitting method to the BHE by solving two nonlinear subproblems. In [14], they propose a non-standard, finite difference scheme to approximate the solution of a generalized Burgers-Huxley equation. Also, in [15] a fourth order finite-difference scheme in a two-time level recurrence relation is proposed for the numerical solution of the generalized Burger-Huxley equation with stability analysis. The resulting nonlinear system is solved by predictorcorrector method. A higher order finite difference scheme [16] and B-spline collocation scheme [17] are implemented to find numerical solution of the generalized BHE.

In this work, we apply the Strang splitting method to the BHE and prove the convergence of the method theoretically in Sobolev spaces and numerically check the results. For the theoretical proof we follow the similar approach to [5] and [11]. Since the linear and nonlinear parts of the BHE have differences we apply the Strang splitting method and solve each subproblems easily. For the convergence analysis, we derive error bounds under given regularity results and properties of the Sobolev spaces. Our main aim is to prove the convergence rates in Sobolev spaces. We solve the BHE by dividing the problem into linear and nonlinear parts without doing any linearization.

In [4], the KdV equation is studied and they apply Lie-Trotter and Strang splitting in order to have error estimates for convergence. They actively make use of the fact that solutions of KdV equation remain bounded in a Sobolev space and this, together with a bootstrap argument guarantees the existence of a uniform choice of time step $\Delta t$ that prevents the solution from blowing up. On the other hand, [5] studies equation with a Burgers type nonlinearity including the KdV equation. They

\footnotetext{
* Corresponding author. Tel.: +90 5073954944.

E-mail address: yesimyazici@iyte.edu.tr, yesim.yazici@yahoo.com (Y. Çiçek).
} 
make use of the fact that solutions of Burgers type equations remain bounded in a Sobolev space and perform an analysis which identifies error terms in the local error as quadrature errors which are estimated via Lie commutator bounds. In [6] and [7], similar analysis are studied for linear evolution equations and for nonlinear Schrödinger equations, respectively. The work is original with respect to the combination of the convergence analysis and computation.

This paper is organized as follows. In Section 2, we give a brief introduction to the Strang splitting method, introduce the model problem (BHE) then split the problem into linear and nonlinear parts. In Section 3, we present hypothesis about the local well-posedness and boundedness of the solution to the BHE then we deal with the regularity results for the BHE. Local and global error analysis in $H^{S}(\mathbb{R})$ are given in Section 4. Finally, we give the numerical results and prove the correct convergence rates for Strang splitting method.

\section{Application the Strang splitting method to the BHE}

The idea of operator splitting [8-10] is widely used for the approximation of partial differential equations. The basic idea is based on splitting a complex problem into simpler sub-problems, each of which is solved by an efficient method. One of the reasons for the popularity of operator splitting is the use of dedicated special numerical techniques for each of the equations.

We focus our attention on the case of linear and nonlinear operators such as,

$$
u_{t}=A u(t)+B(u(t)) \text {, with } t \in[0, T],\left.u\right|_{t=t_{0}}=u_{0}
$$

We employ Lie-Trotter splitting method to the one-dimensional Burgers-Huxley equation,

$$
u_{t}+\alpha u u_{x}-\epsilon u_{x x}=\beta(1-u)(u-\gamma) u,
$$

with the initial condition

$$
\left.u\right|_{t=t_{0}}=u_{0}
$$

where $t>0, \alpha, \beta \geq 0,0<\epsilon \leq 1$ and $0<\gamma<1$. When $\alpha=0$ and $\epsilon=1$, Eq. (2) reduces to Huxley equation and when $\beta=0$, reduces to Burgers' equation.

With the help of the operator splitting, we break (2) into linear diffusion equation and nonlinear reaction equation. In this latter type of the operator splitting, the simpler equations are solved and then recoupled over the initial conditions in delicate ways to preserve a certain accuracy. The exact solution at the time $t$ of $(2)$ is given by $u(t)=\Phi_{A+B}^{t}\left(u_{0}\right)$ with given initial condition and the approximate split solution is denoted by $u_{n}$, at $t=n \Delta t \leq T$, as $\Delta t \rightarrow 0$, where $u_{n+1}=\Phi_{A}^{\Delta t / 2}\left(\Phi_{B}^{\Delta t}\left(\Phi_{A}^{\Delta t / 2}\left(u_{n}\right)\right)\right), n=0,1,2, \ldots$

In our case we split Eq. (2) into two subequations,

$$
v_{t}=A v=\epsilon v_{x x}
$$

and

$$
w_{t}=B(w)=\beta(1-w)(w-\gamma) w-\alpha w w_{x}
$$

acting on appropriate Sobolev spaces.

\section{Error bounds and regularity results for Strang splitting}

In the beginning of the analysis, we assume that the solutions to the BHE are locally well-posed and bounded. Thus, the following hypotheses are about the local well-posedness of the solutions to (2) and boundedness of the solution and initial condition in Sobolev spaces.

Hypothesis 3.0.1. For a fixed time $T$, there exists $M>0$ such that for all $u_{0}$ in $H^{k}(\mathbb{R})$ with $\left\|u_{0}\right\|_{H_{k}} \leq M$, there exists a unique strong solution $u$ in $C\left([0, T], H^{k}\right)$ of $(2)$. In addition, for the initial data $u_{0}$ there exists a constant $K(M, T)<\infty$, such that

$$
\|\tilde{u}(t)-u(t)\|_{H^{k}} \leq K(M, T)\left\|\tilde{u}_{0}-u_{0}\right\|_{H^{k}}
$$

for two arbitrary solutions $u$ and $\tilde{u}$, corresponding to two different initial data $\tilde{u}_{0}$ and $u_{0}$.

Hypothesis 3.0.2. The solution $u(t)$ and the initial data $u_{0}$ of $(2)$ are both in $H^{k}(\mathbb{R})$, and are bounded as

$$
\|u(t)\|_{H^{k}} \leq M<\rho \text { and }\left\|u_{o}\right\|_{H^{k}} \leq C<\infty,
$$

for $0 \leq t \leq T$ and $M<\rho<\infty$.

We define following set of integers such that:

$$
s \geq 1, \quad m=s+3, \quad n=s+1=m-2,
$$

We specify for which integers the hypothesis should hold in the lemmas and theorems for the Strang splitting method. 
3.1. Results for the nonlinear part

We will present and prove several results to estimate the local error for the Strang splitting for the Burgers-Huxley equation. We need to show that there exists a small time step $\Delta t$ for the solutions $\Phi_{A}^{t}\left(v_{0}\right)$ and $\Phi_{B}^{t}\left(w_{0}\right)$ in a Sobolev spaces.

Lemma 1. For $m$ and $n$ in (7) assume the solution $\Phi_{B}^{t}\left(w_{0}\right)=w(t)$ of (5) with initial data $w_{0}$ in $H^{m}(\mathbb{R})$, satisfies $\left\|\Phi_{B}^{t}\left(w_{0}\right)\right\|_{H^{n}} \leq$ $\xi$ for $0 \leq t \leq \Delta t$. Then $\Phi_{B}^{t}\left(w_{0}\right)$ is in $H^{m}(\mathbb{R})$ and in particular

$$
\left\|\Phi_{B}^{t}\left(w_{0}\right)\right\|_{H^{m}} \leq e^{c \xi_{1} t}\left\|w_{0}\right\|_{H^{m}},
$$

where $\xi_{1}=\left(C+2 C \xi+C \xi^{2}\right), C$ is a general constant and $c$ is independent of $w_{0}$.

Proof. From the definition of norm $H^{m}(\mathbb{R})$, we find that $w(t)$ satisfies

$$
\begin{aligned}
\frac{1}{2} \frac{d}{d t}\left\|\Phi_{B}^{t}\left(w_{0}\right)\right\|_{H^{m}}^{2} & \frac{1}{2} \frac{d}{d t}\|w\|_{H^{m}}^{2}=\frac{1}{2} \frac{d}{d t} \sum_{j=0}^{m} \int_{\mathbb{R}} \partial_{x}^{j} w \partial_{x}^{j} w_{t} d x \\
= & \left(w, w_{t}\right)_{H^{m}}=\left(w, \beta w(1-w)(w-\gamma)-\alpha w w_{x}\right)_{H^{m}} \\
= & \beta(1+\gamma) \sum_{j=0}^{m} \sum_{k=0}^{j}\left(\begin{array}{l}
j \\
k
\end{array}\right) \int_{\mathbb{R}} \partial_{x}^{j} w \partial_{x}^{k} w \partial_{x}^{j-k} w d x \\
& -\beta \sum_{j=0}^{m} \sum_{k=0}^{j} \sum_{l=0}^{k}\left(\begin{array}{l}
j \\
k
\end{array}\right)\left(\begin{array}{l}
k \\
l
\end{array}\right) \int_{\mathbb{R}} \partial_{x}^{j} w \partial_{x}^{l} w \partial_{x}^{k-l} w \partial_{x}^{j-k} w d x \\
& -\beta \gamma \sum_{j=0}^{m} \int_{\mathbb{R}} \partial_{x}^{j} w \partial_{x}^{j} w d x-\alpha \sum_{j=0}^{m} \sum_{k=0}^{j}\left(\begin{array}{l}
j \\
k
\end{array}\right) \int_{\mathbb{R}} \partial_{x}^{j} w \partial_{x}^{k+1} w \partial_{x}^{j-k} w
\end{aligned}
$$

We investigate the each part for different cases.

Case 1: For $j<m$ and $k<j$, we obtain for the first term of (9)

$$
\begin{aligned}
& \left|\int_{\mathbb{R}} \partial_{x}^{j} w \partial_{x}^{k} w \partial_{x}^{j-k} w d x\right| \\
& \quad \leq \int_{\mathbb{R}}\left|\partial_{x}^{j} w \partial_{x}^{k} w \partial_{x}^{j-k} w\right| d x \\
& \quad \leq\left\|\partial_{x}^{j} w\right\|_{L^{\infty}}\left\|\partial_{x}^{\max \{k, j-k\}} w\right\|_{L^{2}}\left\|\partial_{x}^{\min \{k, j-k\}} w\right\|_{L^{2}} \\
& \quad \leq C\|w\|_{H^{m}}\|w\|_{H^{m}}\|w\|_{H^{n}} \\
& \quad \leq C \xi\|w\|_{H^{m}}^{2}
\end{aligned}
$$

where we have used Sobolev inequality and the fact that

$$
\begin{aligned}
& \max \{k, j-k\} \leq j+1 \leq m \\
& \min \{k, j-k\} \leq \frac{j}{2} \leq \frac{m}{2}=\frac{s-1}{2}+2 \leq s+1=m-2=n
\end{aligned}
$$

since $m \geq 4$.

For the second term of (9),

$$
\begin{aligned}
& \left|\int_{\mathbb{R}} \partial_{x}^{j} w \partial_{x}^{l} w \partial_{x}^{k-l} w \partial_{x}^{j-k} w d x\right| \\
& \quad \leq \int_{\mathbb{R}}\left|\partial_{x}^{j} w \partial_{x}^{l} w \partial_{x}^{k-l} w \partial_{x}^{j-k} w\right| d x \\
& \quad \leq\left\|\partial_{x}^{j} w\right\|_{L^{\infty}}\left\|\partial_{x}^{j-k} w\right\|_{L^{\infty}} \int_{\mathbb{R}}\left|\partial_{x}^{l} w \partial_{x}^{k-l} w\right| d x \\
& \quad \leq\|w\|_{H^{m}}\|w\|_{H^{m}}\left\|\partial_{x}^{l} w\right\|_{L^{2}}\left\|\partial_{x}^{k-l} w\right\|_{L^{2}} \\
& \quad \leq C\|w\|_{H^{m}}^{2}\|w\|_{H^{m}}\|w\|_{H^{k-m}} \\
& \quad \leq C \xi^{2}\|w\|_{H^{m}}^{2}
\end{aligned}
$$

If we take $l<k \leq n$ and $k-l<n$. 
For the third term of (9),

$$
\begin{aligned}
\left|\int_{\mathbb{R}} \partial_{x}^{j} w \partial_{x}^{j} w d x\right| & \leq \int_{\mathbb{R}}\left|\partial_{x}^{j} w \partial_{x}^{j} w\right| d x \\
& \leq\left\|\partial_{x}^{j} w\right\|_{L^{2}} \mid \partial_{x}^{j} w \|_{L^{2}} \\
& \leq C\|w\|_{H^{m}}^{2}
\end{aligned}
$$

The last term of (9) we have the bound

$$
\begin{aligned}
\left|\int_{\mathbb{R}} \partial_{x}^{j} w \partial_{x}^{k+1} w \partial_{x}^{j-k} w d x\right| & \leq\left\|\partial_{x}^{j} w\right\|_{L^{\infty}}\|w\|_{H^{m}}\|w\|_{H^{n}} \\
& \leq C \xi\|w\|_{H^{m}}^{2}
\end{aligned}
$$

see [11].

Case 2: For $j=m$, we obtain for the first term of (9)

$$
\begin{aligned}
\left|\int_{\mathbb{R}} \partial_{x}^{j} w \partial_{x}^{k} w \partial_{x}^{j-k} w d x\right| & \leq\left\|\partial_{x}^{k} w\right\|_{L^{\infty}}\left\|\partial_{x}^{m} w\right\|_{L^{2}}\left\|\partial_{x}^{m-k} w\right\|_{L^{2}} \\
& \leq C\left\|\partial_{x} w\right\|_{H^{k}}\|w\|_{H^{m}}\|w\|_{H^{m-k}} \\
& \leq C\|w\|_{H^{k+1}}\|w\|_{H^{m}}^{2}
\end{aligned}
$$

To get a bound we investigate this inequality in two cases; when $k+1 \leq n$ and when $k=n$. For the first case we obtain

$$
\left|\int_{\mathbb{R}} \partial_{x}^{j} w \partial_{x}^{k} w \partial_{x}^{j-k} w d x\right| \leq C \xi\|w\|_{H^{m}}^{2}
$$

For the second case, we get

$$
\begin{aligned}
\left|\int_{\mathbb{R}} \partial_{x}^{j} w \partial_{x}^{k} w \partial_{x}^{j-k} w d x\right| & \leq\|w\|_{H^{n+1}}\|w\|_{H^{m}}\|w\|_{H^{m-n}} \\
& \leq C \xi\|w\|_{H^{m}}^{2}
\end{aligned}
$$

here we have used that $n+1 \leq n+2 \leq m$, and $m-n=2 \leq s+1=n$.

We are left with 2 cases; $k \leq m$ and $k=m=j$. For the first case we get,

$$
\begin{aligned}
\left|\int_{\mathbb{R}} \partial_{x}^{j} w \partial_{x}^{k} w \partial_{x}^{j-k} w d x\right| & \leq\left\|\partial_{x}^{m} w\right\|_{L^{2}}\left\|\partial_{x}^{k} w\right\|_{L^{2}}\left\|\partial_{x}^{m-k} w\right\|_{L^{\infty}} \\
& \leq C\|w\|_{H^{m}}\|w\|_{H^{m}}\|w\|_{H^{m-k+1}} \\
& \leq C \xi\|w\|_{H^{m}}^{2}
\end{aligned}
$$

because $m-k+1<m-n \leq 2 \leq n$. For the second case, we have

$$
\begin{aligned}
\left|\int_{\mathbb{R}} \partial_{x}^{m} w \partial_{x}^{m} w w d x\right| & \leq\|w\|_{L^{\infty}}\left\|\partial_{x}^{m} w\right\|_{L^{2}}\left\|\partial_{x}^{m} w\right\|_{L^{2}} \\
& \leq C\|w\|_{H^{n}}\|w\|_{H^{m}}^{2} \\
& \leq C \xi\|w\|_{H^{m}}^{2} .
\end{aligned}
$$

For the second term of (9),

$$
\begin{aligned}
& \left|\int_{\mathbb{R}} \partial_{x}^{m} w \partial_{x}^{l} w \partial_{x}^{k-l} w \partial_{x}^{m-k} w d x\right| \\
& \quad \leq\left\|\partial_{x}^{l} w\right\|_{L^{\infty}}\left\|\partial_{x}^{k-l} w\right\|_{L^{\infty}}\left\|\partial_{x}^{m} w\right\|_{L^{2}}\left\|\partial_{x}^{m-k} w\right\|_{L^{2}} \\
& \quad \leq C\|w\|_{H^{l+1}}\|w\|_{H^{k-l+1}}\|w\|_{H^{m}}\|w\|_{H^{m-k}}
\end{aligned}
$$

The above inequality is divided in two cases; when $l+1 \leq n, k-l+1 \leq n$ and $l+1 \leq n, k=n$. For the first case we have

$$
\begin{aligned}
& \left|\int_{\mathbb{R}} \partial_{x}^{m} w \partial_{x}^{l} w \partial_{x}^{k-l} w \partial_{x}^{m-k} w d x\right| \\
& \quad \leq C\|w\|_{H^{n}}\|w\|_{H^{n}}\|w\|_{H^{m}}\|w\|_{H^{m}} \\
& \quad \leq C \xi^{2}\|w\|_{H^{m}}^{2}
\end{aligned}
$$

For the second case we have

$$
\begin{aligned}
& \left|\int_{\mathbb{R}} \partial_{x}^{m} w \partial_{x}^{l} w \partial_{x}^{k-l} w \partial_{x}^{m-k} w d x\right| \\
& \quad \leq C\|w\|_{H^{n}}\|w\|_{H^{m}}\|w\|_{H^{m}}\|w\|_{H^{n}} \\
& \quad \leq C \xi^{2}\|w\|_{H^{m}}^{2}
\end{aligned}
$$


Since, $n-l+1 \leq m$, and $m-n \leq 2 \leq s+1=n$.

We are left with three cases; $l+1=k=n, l+1 \leq m$, with $m=n$ and $k=m=j=l$. For the first case, we obtain

$$
\begin{aligned}
& \left|\int_{\mathbb{R}} \partial_{x}^{m} w \partial_{x}^{l} w \partial_{x}^{k-l} w \partial_{x}^{m-k} w d x\right| \\
& \quad \leq\left\|\partial_{x}^{m-k} w\right\|_{L^{\infty}}\left\|\partial_{x}^{l} w\right\|_{L^{\infty}}\left\|\partial_{x}^{m} w\right\|_{L^{2}}\left\|\partial_{x}^{k-l} w\right\|_{L^{2}} \\
& \quad \leq C\|w\|_{H^{m-k+1}}\|w\|_{H^{++1}}\|w\|_{H^{m}}\|w\|_{H^{k-l}} \\
& \quad \leq C\|w\|_{H^{m}}\|w\|_{H^{n}}\|w\|_{H^{m}}\|w\|_{H^{n}}
\end{aligned}
$$

Since, $n-l \leq n, \quad m-k+1 \leq m$. For the second case we get the same result, but now we use that $m-k+1 \leq m-n \leq 2 \leq n$.

For the third case,

$$
\begin{aligned}
\left|\int_{\mathbb{R}} \partial_{x}^{m} w \partial_{x}^{m} w w w d x\right| & \leq \int_{\mathbb{R}}\left|\left(\partial_{x}^{m} w\right)^{2} w^{2}\right| d x \leq\|w\|_{L^{\infty}}^{2}\left\|\partial_{x}^{m} w\right\|_{L^{2}}^{2} \\
& \leq C\|w\|_{H^{n}}^{2}\|w\|_{H^{m}}^{2} \\
& \leq C \xi^{2}\|w\|_{H^{m}}^{2}
\end{aligned}
$$

For the third term of (9),

$$
\begin{aligned}
\left|\int_{\mathbb{R}} \partial_{x}^{m} w \partial_{x}^{m} w d x\right| & \leq\left\|\partial_{x}^{m} w\right\|_{L^{2}}\left\|\partial_{x}^{m} w\right\|_{L^{2}} \\
& \leq C\|w\|_{H^{m}}^{2}
\end{aligned}
$$

Finally, the last term of (9) we have the bound

$$
\left|\int_{\mathbb{R}} \partial_{x}^{m} w \partial_{x}^{k+1} w \partial_{x}^{m-k} w d x\right| \leq C \xi^{2}\|w\|_{H^{m}}^{2}
$$

see [11].

All in all we get, by summing up the estimates, the following inequality

$$
\frac{d}{d t}\|w(t)\|_{H^{m}}^{2}=\|w(t)\|_{H^{m}} \frac{d}{d t}\|w(t)\|_{H^{m}} \leq c \xi_{1}\|w(t)\|_{H^{m}}^{2}
$$

which leads to

$$
\frac{d}{d t}\|w(t)\|_{H^{m}} \leq c \xi_{1}\|w(t)\|_{H^{m}}
$$

where $\xi_{1}=\left(C+2 C \xi+C \xi^{2}\right)$. This result concludes the proof [5].

Lemma 2. Assume $\left\|w_{0}\right\|_{H^{k}} \leq K$ for some $k \geq 1$. Then there exists $\bar{t}(K)>0$ such that $\left\|\Phi_{B}^{t}\left(w_{0}\right)\right\|_{H^{k}} \leq 2 K$ for $0 \leq t \leq \bar{t}(K)$.

Proof. By doing the same calculations as in the proof of Lemma 1 with $k$ instead of $m$ and using the bound for $u_{0}$ in $H^{k}(\mathbb{R})$, we arrive with the following inequality

$$
\|w(t)\|_{H^{k}} \frac{d}{d t}\|w(t)\|_{H^{k}} \leq c\|w(t)\|_{H^{k}}^{4}
$$

which simplifies to

$$
\frac{d}{d t}\|w(t)\|_{H^{k}} \leq c\|w(t)\|_{H^{k}}^{3}
$$

The result follows by comparing with the solution of the differential equation $y^{\prime}=c y^{3}$.

Lemma 3. If $\left\|w_{0}\right\|_{H^{s+2}} \leq C_{0}$ for $s \geq 1$, then there exists $\bar{t}$ depending on $C_{0}$, such that the solution $w(t)$ of $(5)$ is $C^{3}\left([0, \bar{t}], H^{s}\right)$.

Proof. Let $t$ be in $[0, \bar{t}]$, with $\bar{t}$ from Lemma 2 , and define

$$
\tilde{w}(t)=w_{0}+t B\left(w_{0}\right)+\int_{0}^{t}(t-s) d B(w(s))[B(w(s))] d s,
$$

where $d B().[$.$] is the Fréchet derivative. Calculating the second derivative of \tilde{w}$, gives

$$
\begin{aligned}
\tilde{w}_{t t}= & d B(w(s))[B(w(s))] \\
= & -3 \beta w^{2}\left(-w^{3}+(1+\gamma) w^{2}-\gamma w\right)+3 \alpha w^{2} w w_{x} \\
& +2 \beta(1+\gamma) w\left(-w^{3}+(1+\gamma) w^{2}-\gamma w\right)-2 \alpha(1+\gamma) w w_{x} \\
& -\gamma \beta\left(-w^{3}+(1+\gamma) w^{2}-\gamma w\right)+\gamma \alpha w w_{x} \\
& -\beta w\left(-3 w^{2} w_{x}+2(1+\gamma) w w_{x}-\gamma w_{x}\right)+\alpha\left(w w_{x}^{2}+w^{2} w_{x x}\right) \\
& -\beta w_{x}\left(-w^{3}+(1+\gamma) w^{2}-\gamma w\right)+\alpha w w_{x}^{2}
\end{aligned}
$$


By differentiation (5) with respect to $t$, we get

$$
\begin{aligned}
w_{t t} & =B(w)_{t}=\left(-\beta w^{3}+\beta(1+\gamma) w^{2}-\beta \gamma w-\alpha w w_{x}\right)_{t} \\
& =-3 \beta w^{2} w_{t}+2 \beta(1+\gamma) w w_{t}-\beta \gamma w_{t}-\alpha w_{t} w_{x}-\alpha w w_{x t} \\
& =-3 \beta w^{2}(B(w))+2 \beta(1+\gamma) w(B(w))-\beta \gamma(B(w))-\alpha(B(w)) w_{x}-\alpha w(B(w))_{x} \\
& =\tilde{w}_{t t}
\end{aligned}
$$

we see that $\tilde{w}(0)=u_{0}$ and $\tilde{w}_{t}(0)=B\left(u_{0}\right)=w_{t}$. Thus we have shown that $w=\tilde{w}$. The same is also true for $\tilde{w_{t t t}}$. It follows that $\tilde{w}$ is $C^{3}\left([0, \bar{t}], H^{s}\right)$.

\section{Local error in $\boldsymbol{H}^{s}$ space}

Lemma 4. Let $s \geq 1$ be an integer and Hypothesis 3.0.2 holds for $k=m$ for the solution $u(t)=\Phi_{A+B}^{\Delta t}\left(u_{0}\right)$ of (2). If the initial data $u_{0}$ is in $H^{m}(\mathbb{R})$, then the local error of the Strang splitting is bounded in $H^{s}(\mathbb{R})$ by

$$
\left\|\Phi_{A}^{\Delta t / 2}\left(\Phi_{B}^{\Delta t}\left(\Phi_{A}^{\Delta t / 2}\left(u_{0}\right)\right)\right)-\Phi_{A+B}^{\Delta t}\left(u_{0}\right)\right\|_{H^{s}} \leq c_{s} \Delta t^{3},
$$

where $c_{S}$ only depends on $\left\|u_{0}\right\|_{H^{m}}$.

Proof. We start with

$$
B(\varphi(s))-B(\varphi(0))=\int_{0}^{s} d B(\varphi(\rho))[\dot{\varphi}(\rho)] d \rho
$$

where

$$
\begin{aligned}
& \varphi(\rho)=\Phi_{A}^{(s-\rho)}(u(\rho)), \\
& \dot{\varphi}(\rho)=\Phi_{A}^{(s-\rho)}(B(u(\rho))) .
\end{aligned}
$$

Hence we get,

$$
B(u(s))=B\left(\Phi_{A}^{s}\left(u_{0}\right)\right)+\int_{0}^{s} d B\left(\Phi_{A}^{(s-\rho)}(u(\rho))\right)\left[\Phi_{A}^{(s-\rho)}(B(u(\rho)))\right] d \rho,
$$

where we have used that $\varphi(0)=\Phi_{A}^{s}(u(0))=\Phi_{A}^{s}\left(u_{0}\right)$. From the variation of constants formula we have the exact solution of (2) such that,

$$
\Phi_{A+B}^{t}\left(u_{0}\right)=\Phi_{A}^{t}\left(u_{0}\right)+\int_{0}^{t} \Phi_{A}^{(t-s)}(B(u(s))) d s
$$

To find the exact solution after one step, we insert (37) into (38) and get the following:

$$
u(\Delta t)=\Phi_{A}^{\Delta t}\left(u_{0}\right)+\int_{0}^{\Delta t} \Phi_{A}^{(\Delta t-s)}\left(B\left(\Phi_{A}^{s}\left(u_{0}\right)\right)\right) d s+\int_{0}^{\Delta t} \int_{0}^{s} \Phi_{A}^{(\Delta t-s)}(H(u(\rho))) d \rho d s,
$$

where $H(u(\rho))$ is defined for a general vector $v$ as

$$
H(v)=d B\left(\Phi_{A}^{(s-\rho)} v\right)\left[\Phi_{A}^{(s-\rho)} B(v)\right]
$$

Using (37), we get

$$
H(u(\rho)))=H\left(\Phi_{A}^{\rho}\left(u_{0}\right)\right)+\int_{0}^{\rho} d H\left(\Phi_{A}^{(\rho-\tau)}(u(\tau))\right)\left[\Phi_{A}^{(\rho-\tau)}(B(u(\tau)))\right] d \tau,
$$

where

$$
d H(v)[w]=d^{2} B\left(\Phi_{A}^{(s-\rho)}(v)\right)\left[\Phi_{A}^{(s-\rho)}(w), \Phi_{A}^{(s-\rho)}(B(v))\right]+d B\left(\Phi_{A}^{(s-\rho)}(v)\right)\left[\Phi_{A}^{(s-\rho)}(d B(v)[w])\right] .
$$

Substituting the integral formula for $H$ into (39) we get,

$$
u(\Delta t)=\Phi_{A}^{\Delta t}\left(u_{0}\right)+\int_{0}^{\Delta t} \Phi_{A}^{(\Delta t-s)}\left(B\left(\Phi_{A}^{s}\left(u_{0}\right)\right)\right) d s+S 1
$$

where

$$
\begin{aligned}
S 1= & \int_{0}^{\Delta t} \int_{0}^{s} \Phi_{A}^{(\Delta t-s)}\left(d B\left(\Phi_{A}^{s}\left(u_{0}\right)\right)\left[\Phi_{A}^{(s-\rho)}\left(B\left(\Phi_{A}^{s}\left(u_{0}\right)\right)\right)\right]\right) d \rho d s \\
& +\int_{0}^{\Delta t} \int_{0}^{s} \int_{0}^{\rho} d H\left(\Phi_{A}^{(\rho-\tau)}(u(\tau))\right)\left[\Phi_{A}^{(\rho-\tau)}(B(u(\tau)))\right] d \tau d \rho d s .
\end{aligned}
$$


One step with Strang splitting is

$$
u_{1}=\Phi_{A}^{\Delta t / 2}\left(\Phi_{B}^{\Delta t}\left(\Phi_{A}^{\Delta t / 2}\left(u_{0}\right)\right) .\right.
$$

We can write the second order Taylor expansion for the $\Phi_{B}^{\Delta t}$ as follows:

$$
\begin{aligned}
\Phi_{B}^{\Delta t}(v)= & v+\Delta t B(v)+\frac{1}{2} \Delta t^{2} d B(v)[B(v)]+(\Delta t)^{3} \int_{0}^{1} \frac{1}{2}(1-\theta)^{2}\left(d^{2} B\left(\Phi_{B}^{\theta \Delta t}(v)\right)\left[B\left(\Phi_{B}^{\theta \Delta t}(v)\right), B\left(\Phi_{B}^{\theta \Delta t}(v)\right)\right]\right. \\
& \left.+d B\left(\Phi_{B}^{\theta \Delta t}(v)\right)\left[d B\left(\Phi_{B}^{\theta \Delta t}(v)\right)\left[B\left(\Phi_{B}^{\theta \Delta t}(v)\right)\right]\right]\right) d \theta
\end{aligned}
$$

After simplification the notation we rewrite the integrand as

$$
\Phi_{B}^{\Delta t}(v)=v+\Delta t B(v)+\frac{1}{2} \Delta t^{2} d B(v)[B(v)]+(\Delta t)^{3} \int_{0}^{1} \frac{1}{2}(1-\theta)^{2}\left(d^{2} B(B, B)+d B d B B\right)\left(\Phi_{B}^{\theta \Delta t}(v)\right) d \theta
$$

Inserting this series expansion into (45), we obtain

$$
u_{1}=\Phi^{\Delta t A} u_{0}+\Delta t \Phi_{A}^{\Delta t / 2}\left(B\left(\Phi_{A}^{\Delta t / 2}\left(u_{0}\right)\right)\right)+\frac{1}{2} \Delta t^{2} \Phi_{A}^{\Delta t / 2}\left(d B\left(\Phi_{A}^{\Delta t / 2}\left(u_{0}\right)\right)\left[B\left(\Phi_{A}^{\Delta t / 2}\left(u_{0}\right)\right)\right]\right)+S 2
$$

where

$$
S 2=\Delta t^{3} \int_{0}^{1} \frac{1}{2}(1-\theta)^{2} \Phi_{A}^{\Delta t / 2}\left(d^{2} B(B, B)+d B d B B\right)\left(\Phi_{B}^{\theta \Delta t}\left(\Phi_{A}^{\Delta t / 2}\left(u_{0}\right)\right)\right) d \theta .
$$

The local error after one step is,

$$
\begin{aligned}
u_{1}-u(\Delta t)= & \Delta t \Phi_{A}^{\Delta t / 2}\left(B\left(\Phi_{A}^{\Delta t / 2}\left(u_{0}\right)\right)\right)-\int_{0}^{\Delta t} \Phi_{A}^{(\Delta t-s)}\left(B\left(\Phi_{A}^{s}(u(s))\right)\right) d s \\
& +\frac{1}{2}(\Delta t)^{2} \Phi_{A}^{\Delta t / 2}\left(d B\left(\Phi_{A}^{\Delta t / 2}\left(u_{0}\right)\right)\left[B\left(\Phi_{A}^{\Delta t / 2}\left(u_{0}\right)\right)\right]\right)+(S 2-S 1) .
\end{aligned}
$$

We can rewrite the above expression in a simpler form by defining,

$$
\begin{aligned}
& h(s, \rho)=\Phi_{A}^{(\Delta t-s)}\left(d B\left(\Phi_{A}^{s}\left(u_{0}\right)\right)\left[\Phi_{A}^{(s-\rho)}\left(B\left(\Phi_{A}^{s}\left(u_{0}\right)\right)\right)\right]\right), \\
& f(s)=\Phi_{A}^{(\Delta t-s)}\left(B\left(\Phi_{A}^{s}\left(u_{0}\right)\right)\right)
\end{aligned}
$$

Hence we can write the local error in a simplified form,

$$
u_{1}-u(\Delta t)=\Delta t f(\Delta t / 2)-\int_{0}^{\Delta t} f(s) d s+\frac{1}{2}(\Delta t)^{2} h(\Delta t / 2, \Delta t / 2)-\int_{0}^{\Delta t} \int_{0}^{s} h(s, \rho) d \rho d s+S 3-S 4
$$

where

$$
S 3=(\Delta t)^{3} \int_{0}^{1} \frac{1}{2}(1-\theta)^{2} \Phi_{A}^{\Delta t / 2}\left(d^{2} B(B, B)+d B d B B\right)\left(\Phi_{B}^{\theta \Delta t}\left(\Phi_{A}^{\Delta t / 2}\left(u_{0}\right)\right)\right) d \theta
$$

and

$$
S 4=\int_{0}^{\Delta t} \int_{0}^{s} \int_{0}^{\rho} d H\left(\Phi_{A}^{(\rho-\tau)}(u(\tau))\right)\left[\Phi_{A}^{(\rho-\tau)}(B(u(\tau)))\right] d \tau d \rho d s .
$$

The difference of the first two terms is the error of the midpoint rule and the second line of (53) is the error of the two dimensional quadrature rule. We can rewrite Eq. (53) by using these error rules and triangle rule

$$
\left\|u_{1}-u(\Delta t)\right\|_{H^{s}} \leq \int_{0}^{\Delta t}\left\|k(t) f^{\prime \prime}(t)\right\|_{H^{s}} d s+\left\|\frac{1}{2}(\Delta t)^{2} h(\Delta t / 2, \Delta t / 2)-\int_{0}^{\Delta t} \int_{0}^{s} h(s, \rho) d \rho d s\right\|_{H^{s}}+\|S 3\|_{H^{s}}+\|S 4\|_{H^{s}}
$$

where $k(t)$ is bounded kernel and $f^{\prime \prime}(t)$ is the Fréchet derivative given as

$$
\begin{aligned}
f^{\prime \prime}(s)= & \Phi_{A}^{(\Delta t-s)}\left((d A(v))^{2}[B(v)]-d A(v)[d B(v)[A(v)]]-d^{2} A(v)[B(v), A(v)]-d A(v)[d B(v)[A(v)]]\right. \\
& \left.+d^{2} B(v)[A(v)]^{2}+d B(v)[d A(v)[A(v)]]\right) .
\end{aligned}
$$

We know from the linearity of the flow of $A$ such that $\Phi_{A}^{(\Delta t-s)}$ is bounded in Sobolev norm. We find the Fréchet derivatives of the given operators $A$ and $B$ in (4) and (5),

$$
\begin{aligned}
d A(v)[h] & =A(h) \\
d^{2} A(v)[h, k] & =0 \\
d B(v)[h] & =-3 \beta v^{2} h+2 \beta(1+\gamma) v h-\beta \gamma h-\alpha(v h)_{x} \\
d^{2} B(v)[h, k] & =-6 \beta v k h+2 \beta(1+\gamma) k h-\alpha(k h)_{x}
\end{aligned}
$$


By using these derivatives of the operators $A$ and $B$ we get the following:

$$
\begin{aligned}
f^{\prime \prime}(s)= & -\partial_{x}^{4} \beta v^{3}+\beta(1+\gamma) \partial_{x}^{4} v^{2}+\partial_{x}^{4}\left(-3 \beta v^{2}+2 \beta \gamma(1+\gamma)-4 v+2-\gamma\right) \\
& +6 \partial_{x}^{2}\left(v^{2} \partial_{x}^{2} v\right)-4(1+\gamma) \partial_{x}^{2}\left(v v_{x}\right)+\alpha \partial_{x}^{4}\left(v v_{x}\right) \\
& -2 \alpha \partial_{x}^{2}\left(\left(v \partial_{x}^{2}(v)\right)_{x}\right)+\left(\left(\partial_{x}^{2}(v)\right)^{2}\right)_{x}+\left(v \partial_{x}^{4}(v)\right)_{x}
\end{aligned}
$$

Writing out the argument using the Leibniz' rule gives

$$
\begin{aligned}
\left\|f^{\prime \prime}(s)\right\|_{H^{s}} \leq & \beta \sum_{k=1}^{4}\left(\begin{array}{l}
4 \\
k
\end{array}\right)\left\|\partial_{x}^{k} v^{2} \partial_{x}^{4-k} v\right\|_{H^{s}}+(1+\gamma) \beta \sum_{k=1}^{4}\left(\begin{array}{l}
4 \\
k
\end{array}\right)\left\|\partial_{x}^{k} v \partial_{x}^{4-k} v\right\|_{H^{s}} \\
& +6 \beta \sum_{k=1}^{2}\left(\begin{array}{l}
2 \\
k
\end{array}\right)\left\|\partial_{x}^{k} v^{2} \partial_{x}^{2-k} v\right\|_{H^{s}}+4(1+\gamma) \sum_{k=1}^{2}\left(\begin{array}{l}
2 \\
k
\end{array}\right)\left\|\partial_{x}^{k} v \partial_{x}^{2-k} v\right\|_{H^{s}} \\
& +\sum_{k=1}^{2}\left(\begin{array}{l}
4 \\
k
\end{array}\right)\left\|\partial_{x}^{4-k} v \partial_{x}^{k+1} v\right\|_{H^{s}}+2 \sum_{k=0}^{1}\left(\begin{array}{l}
3 \\
k
\end{array}\right)\left\|\partial_{x}^{3-k} v \partial_{x}^{2+k} v\right\|_{H^{s}} \\
& +(4+\gamma)\left\|\partial_{x}^{4} v\right\|_{H^{s}}+\left\|2 \partial_{x}^{2} v \partial_{x}^{3} v\right\|_{H^{s}} .
\end{aligned}
$$

Using the Banach algebra property and $v=\Phi_{A}^{S} u_{0}$ we get,

$$
\left\|f^{\prime \prime}(s)\right\|_{H^{s}} \leq C\left\|\Phi_{A}^{s} u_{0}\right\|_{H^{p}}^{3} \leq C\left\|u_{0}\right\|_{H^{p}}^{3} .
$$

The bound for the first term of (56) is obtained as,

$$
\begin{aligned}
\int_{0}^{\Delta t}\left\|k(t) f^{\prime \prime}(t)\right\|_{H^{s}} d s & \leq(\Delta t)^{3} \int_{0}^{1}\left\|k(\theta) f^{\prime \prime}(\theta \Delta t)\right\|_{H^{s}} d \theta \\
& \leq(\Delta t)^{3} \int_{0}^{1}\left\|f^{\prime \prime}(\theta \Delta t)\right\|_{H^{s}} d \theta \leq C\left\|u_{0}\right\|_{H^{p}}^{3}(\Delta t)^{3} .
\end{aligned}
$$

For the second line of (56) we use the two dimensional quadrature formula and get the following result:

$$
\left\|\frac{1}{2} \Delta t^{2} h(\Delta t / 2, \Delta t / 2)-\int_{0}^{\Delta t} \int_{0}^{s} h(s, \rho) d \rho d s\right\|_{H^{s}} \leq C \Delta t^{3}\left(\max \left\|\frac{\partial h}{\partial s}\right\|_{H^{s}}+\max \left\|\frac{\partial h}{\partial \rho}\right\|_{H^{s}}\right) .
$$

We need to find the bound for the partial derivatives of $h$. Let us define the following equalities:

$$
\begin{aligned}
v(s) & =\Phi_{A}^{s}\left(u_{0}\right) \\
w(s, \rho) & =\Phi_{A}^{(s-\rho)}(B(v(\rho))) .
\end{aligned}
$$

Now we can write,

$$
h(s, \rho)=\Phi_{A}^{(\Delta t-s)}(d B(v(s))[w(s, \rho)]) .
$$

Start with the first derivative,

$$
\begin{aligned}
\left\|\frac{\partial h}{\partial s}\right\|_{H^{s}}= & \left\|\Phi_{A}^{(\Delta t-s)}\left(-A(d B(v)[w])+d^{2} B(v)[A(v), w]+d B(v)[A(w)]\right)\right\|_{H^{s}} \\
\leq & \left\|-A(d B(v)[w])+d^{2} B(v)[A(v), w]+d B(v)[A(w)]\right\|_{H^{s}} \\
\leq & \left\|-A \beta\left(-3 v^{2} w\right)-6 \beta v A(v) w-3 \beta v^{2} A(w)\right\|_{H^{s}} \\
& +\|-A(2 \beta(1+\gamma) v w)+2 \beta(1+\gamma) A(v) w+2 \beta(1+\gamma) v A(w)\|_{H^{s}} \\
& +\left\|A(v w)_{x}-(A(v) w)_{x}-(v A(w))_{x}\right\|
\end{aligned}
$$

By using the Fréchet derivative and Leibniz' rule we get the following result:

$$
\begin{aligned}
\left\|\frac{\partial h}{\partial s}\right\|_{H^{s}} & \leq C_{1}\left\|u_{0}\right\|_{H^{s+3}}^{3}+C_{2}\left\|u_{0}\right\|_{H^{s+3}}^{4} \\
& \leq C\left\|u_{0}\right\|_{H^{m}}^{4}
\end{aligned}
$$

Now for the other derivative we use the similar approach to previous one and get the result,

$$
\begin{aligned}
\left\|\frac{\partial h}{\partial \rho}\right\| & =\left\|\Phi_{A}^{(\Delta t-s)}\left(d B(v)\left[\Phi_{A}^{(s-\rho)}(-A(B(v))+d B(v)[A(v)])\right]\right)\right\|_{H^{s}} \\
& \leq C\left\|u_{0}\right\|_{H^{m}}^{5} .
\end{aligned}
$$


For the third term in (56) we use the triangle inequality and definition of the second order Fréchet derivative of the operator $B$ which is defined in (5),

$$
\begin{aligned}
\|S 3\|_{H^{s}} & \leq\left\|(\Delta t)^{3} \int_{0}^{1} \Phi_{A}^{\Delta t / 2}\left(d^{2} B(B, B)+d B d B B\right)\left(\Phi_{B}^{\theta \Delta t}\left(\Phi_{A}^{\Delta t / 2}\left(u_{0}\right)\right)\right)\right\|_{H^{s}} d \theta, \\
& \leq(\Delta t)^{3} \int_{0}^{1}\left\|d^{2} B(B, B)(w)+d B d B B(w)\right\|_{H^{s}} \\
& \leq(\Delta t)^{3}\left(\left\|d^{2} B(B, B)(w)\right\|_{H^{s}}+\|d B d B B(w)\|_{H^{s}}\right)
\end{aligned}
$$

where $w$ is redefined as follows:

$$
w=\Phi_{B}^{\theta \Delta t}\left(\Phi_{A}^{\Delta t / 2}\left(u_{0}\right)\right) .
$$

The bound for the first term is obtained easily,

$$
\left\|d^{2} B(B, B)(w)\right\|_{H^{s}} \leq C\left\|u_{0}\right\|_{H^{m}}^{7}
$$

and the second term can be bounded as

$$
\|d B d B B(w)\|_{H^{s}} \leq C\left\|u_{0}\right\|_{H^{m}}^{8}
$$

Hence by using these two results, we get

$$
\|S 3\|_{H^{s}} \leq C(\Delta t)^{3}\left\|u_{0}\right\|_{H^{m}}^{8} \leq C(\Delta t)^{3} .
$$

For the last term in (56) we get,

$$
\|S 4\|_{H^{s}} \leq \int_{0}^{\Delta t} \int_{0}^{s} \int_{0}^{\rho}\|d H(v)[w]\|_{H^{s}} d \tau d \rho d s
$$

where $H$ is defined in (40) and we redefine $v$ and $w$ as follows:

$$
v=\Phi_{A}^{(\rho-\tau)}(u(\tau)) \text { and } w=\Phi_{A}^{(\rho-\tau)}(B(u(\tau))) .
$$

We need to find a bound for the integrand,

$$
\|d H(v)[w]\|_{H^{s}} \leq\left\|d^{2} B\left(\Phi_{A}^{(s-\rho)}(v)\right)\left[\Phi_{A}^{(s-\rho)}(w), \Phi_{A}^{(s-\rho)}(B(v))\right]\right\|_{H^{s}}+\left\|d B\left(\Phi_{A}^{(s-\rho)}(v)\right)\left[\Phi_{A}^{(s-\rho)}(d B(v)[w])\right]\right\|_{H^{s}} .
$$

For the first term, we find a bound by using the same technique as the previous one and get the following estimate:

$$
\begin{aligned}
\| d^{2} B & \left(\Phi_{A}^{(s-\rho)}(v)\right)\left[\Phi_{A}^{(s-\rho)}(w), \Phi_{A}^{(s-\rho)}(B(v))\right] \|_{H^{s}} \\
\leq & \left\|-6 \Phi_{A}^{(s-\rho)}(v)\left(\Phi_{A}^{(s-\rho)}(w) \Phi_{A}^{(s-\rho)}(B(v))\right)\right\|_{H^{s}} \\
& +2(1+\gamma)\left\|\Phi_{A}^{(s-\rho)}(w) \Phi_{A}^{(s-\rho)}(B(v))\right\|_{H^{s}}+\left\|\left(\Phi_{A}^{(s-\rho)}(w) \Phi_{A}^{(s-\rho)}(B(v))\right)_{x}\right\|_{H^{s}}
\end{aligned}
$$

We find the following by using the definition of the operator $B$ :

$$
\begin{aligned}
& \left\|d^{2} B\left(\Phi_{A}^{(s-\rho)}(v)\right)\left[\Phi_{A}^{(s-\rho)}(w), \Phi_{A}^{(s-\rho)}(B(v))\right]\right\|_{H^{s}} \\
& \quad \leq C\left(\left\|u_{0}\right\|_{H^{m}}^{3}+\left\|u_{0}\right\|_{H^{m}}^{4}\right)
\end{aligned}
$$

and the second term is bounded by,

$$
\left\|d B\left(\Phi_{A}^{(s-\rho)}(v)\right)\left[\Phi_{A}^{(s-\rho)}(d B(v)[w])\right]\right\|_{H^{s}} \leq C\left\|u_{0}\right\|_{H^{m}}^{5}
$$

Using (78) and (79) we obtain the following result:

$$
\|S 4\|_{H^{s}} \leq \int_{0}^{\Delta t} \int_{0}^{s} \int_{0}^{\rho} C\left(\left\|u_{0}\right\|_{H^{m}}^{3}+\left\|u_{0}\right\|_{H^{m}}^{4}+\left\|u_{0}\right\|_{H^{m}}^{5}\right) d \tau d \rho d s \leq C(\Delta t)^{3} .
$$

Finally, we get the local error in (56) and this completes the proof.

\section{Global error in $\boldsymbol{H}^{s}$ space}

Theorem 1. Assume there exists a solution of (2). If Hypothesis 3.0.1 holds for $k=s+1$ and Hypothesis 3.0.2 holds for $k=s+3$ then there exists $\bar{\Delta} t>0$ such that for all $\Delta t \leq \overline{\Delta t}$.

$$
\left\|u_{n}-u\left(\cdot, t_{n}\right)\right\|_{H^{s}} \leq C_{s}(\Delta t)^{2}
$$

where $u_{n}$ is Strang splitting solution and $\bar{\Delta} t$ and $C_{s}$ only depends on $\left\|u_{0}\right\|_{H^{s+3}}, \rho$ and $T$ where $T \geq n \Delta t$.

Proof. To prove the global error in $H^{s}(\mathbb{R})$ we use the local error estimate and results for linear and nonlinear parts of the BHE. The proof relies on the induction argument. 
We start with assuming that Hypothesis 3.0.1 and 3.0.2 hold for $k=s$. We use the same notation as in [5], we take

$$
u_{n}^{k}=\Phi_{A+B}^{(n-k) \Delta t}\left(u_{k}\right)=\Phi^{(n-k) \Delta t}\left(u_{k}\right)
$$

as the exact solution to (2) and we make the induction hypothesis that for $k \leq n-1$,

$$
\begin{aligned}
& \left\|u_{k}\right\|_{H^{s}} \leq M \\
& \left\|u_{k}\right\|_{H^{s+3}} \leq C_{1} \\
& \left\|u_{k}-u\left(t_{k}\right)\right\|_{H^{s}} \leq k \Delta t
\end{aligned}
$$

holds for all $k \leq n-1$. We need to show that the above inequalities are true for $k=n$ where $C_{1}$ is a constant from Lemma 1 and $k=K(M, T) c_{S}\left(C_{1}\right)$ where $K(M, T)$ is given in (6) and $c_{S}\left(C_{1}\right)$ is a constant from Lemma 4. Using the telescope sum and the triangle inequality, we write the error as follows:

$$
\left\|u_{n}-u\left(t_{n}\right)\right\|_{H^{s}}=\left\|\sum_{k=0}^{n-1} u_{n}^{k+1}-u_{n}^{k}\right\|_{H^{s}} \leq \sum_{k=0}^{n-1}\left\|u_{n}^{k+1}-u_{n}^{k}\right\|_{H^{s}},
$$

by using the notation we get,

$$
\left\|u_{n}-u\left(\cdot, t_{n}\right)\right\|_{H^{s}} \leq \sum_{k=0}^{n-1} \| \Phi^{(n-k-1) \Delta t}\left(\Psi^{\Delta t}\left(u_{k}\right)-\left(\Phi^{\Delta t}\left(u_{k}\right)\right) \|_{H^{s}}\right.
$$

For $k \leq n-2$ we get by using Hypothesis 3.0.2,

$$
\left\|\Psi^{\Delta t}\left(u_{k}\right)\right\|_{H^{s}}=\left\|u_{k+1}\right\|_{H^{s}} \leq M
$$

where $\Psi^{\Delta t}\left(u_{k}\right)=\Phi_{A}^{\Delta t / 2}\left(\Phi_{B}^{\Delta t}\left(\Phi_{A}^{\Delta t / 2}\left(u_{k}\right)\right)\right)$ and the exact solution,

$$
\left\|\Phi^{\Delta t}\left(u_{k}\right)\right\|_{H^{s}} \leq\left\|\Phi^{\Delta t}\left(u_{k}\right)-\Phi^{\Delta t}\left(u\left(t_{k}\right)\right)\right\|_{H^{s}}+\left\|\Phi^{\Delta t}\left(u\left(t_{k}\right)\right)\right\|_{H^{s}},
$$

by using (6) we get,

$$
\begin{aligned}
& \left\|\Phi^{\Delta t}\left(u_{k}\right)\right\|_{H^{s}} \leq K(M, T)\left\|u_{k}-u\left(t_{k}\right)\right\|_{H^{s}}+\left\|u\left(t_{k+1}\right)\right\|_{H^{s}} \\
& \leq K(M, T) k \Delta t+\rho \leq M
\end{aligned}
$$

Hence using Hypothesis 3.0.1 and the results in (88) and (90) for $k \leq n-1$ we obtain,

$$
\left\|\Phi^{(n-k-1) \Delta t}\left(\Psi^{\Delta t}\left(u_{k}\right)-\Phi^{\Delta t}\left(u_{k}\right)\right)\right\|_{H^{s}} \leq K(M, T) c_{s}\left(C_{1}\right)(\Delta t)^{3} .
$$

Summing up all terms and using $n \Delta t=T$,

$$
\left\|u_{n}-u\left(t_{n}\right)\right\|_{H^{s}} \leq n K(M, T) c_{s}\left(C_{1}\right)(\Delta t)^{3} \leq k(\Delta t)^{3} .
$$

We also need to prove the boundedness $u_{n}$. If we choose $k \Delta t \leq M-\rho$ and use Hypothesis 3.0.2,

$$
\left\|u_{n}\right\|_{H^{s}}=\left\|u_{n}-u\left(t_{n}\right)\right\|_{H^{s}}+\left\|u\left(t_{n}\right)\right\|_{H^{s}} \leq M-\rho+\rho \leq M .
$$

To show that $u_{n}$ is bounded in $H^{s+3}(\mathbb{R})$, we write

$$
\left\|u_{n}\right\|_{H^{s+3}}=\left\|\Phi_{A}^{\Delta t / 2} \circ \Phi_{B}^{\Delta t} \circ \Phi_{A}^{\Delta t / 2}\left(u_{n-1}\right)\right\|_{H^{s+3}} \leq\left\|\Phi_{B}^{\Delta t}\left(u_{n-1}\right)\right\|_{H^{s+3}},
$$

where we have used the boundedness of the linear solution and Lemma 2 such that $\left\|\Phi_{B}^{\Delta t}\left(u_{n-1}\right)\right\|_{H^{s+3}} \leq 2 M$ as long as $\left\|u_{n-1}\right\|_{H^{s+3}}$ is bounded. Thus using Lemma 1 we get the following result:

$$
\left\|u_{n}\right\|_{H^{s+3}} \leq e^{2 \alpha_{1} M \Delta t}\left\|u_{n-1}\right\|_{H^{s+3}} \leq C .
$$

Hence we get the following result:

$$
\begin{aligned}
\left\|u_{n}-u\left(\cdot, t_{n}\right)\right\|_{H^{s}} & \leq \sum_{k=0}^{n-1} \| \Phi^{(n-k-1) \Delta t}\left(\Psi^{\Delta t}\left(u\left(t_{k}\right)\right)-\left(\Phi^{\Delta t}\left(u\left(t_{k}\right)\right)\right) \|_{H^{s}}\right. \\
& \leq \sum_{k=0}^{n-1} K(M, T)\left\|\Psi^{\Delta t}\left(u\left(t_{k}\right)\right)-\Phi^{\Delta t}\left(u\left(t_{k}\right)\right)\right\| \\
& \leq n K(M, T) c_{1}\left(C_{0}\right)(\Delta t)^{3} \\
& \leq T K(M, T) c_{1}\left(C_{0}\right)(\Delta t)^{2} \\
& \leq C(\Delta t)^{2}
\end{aligned}
$$

This completes the proof. 


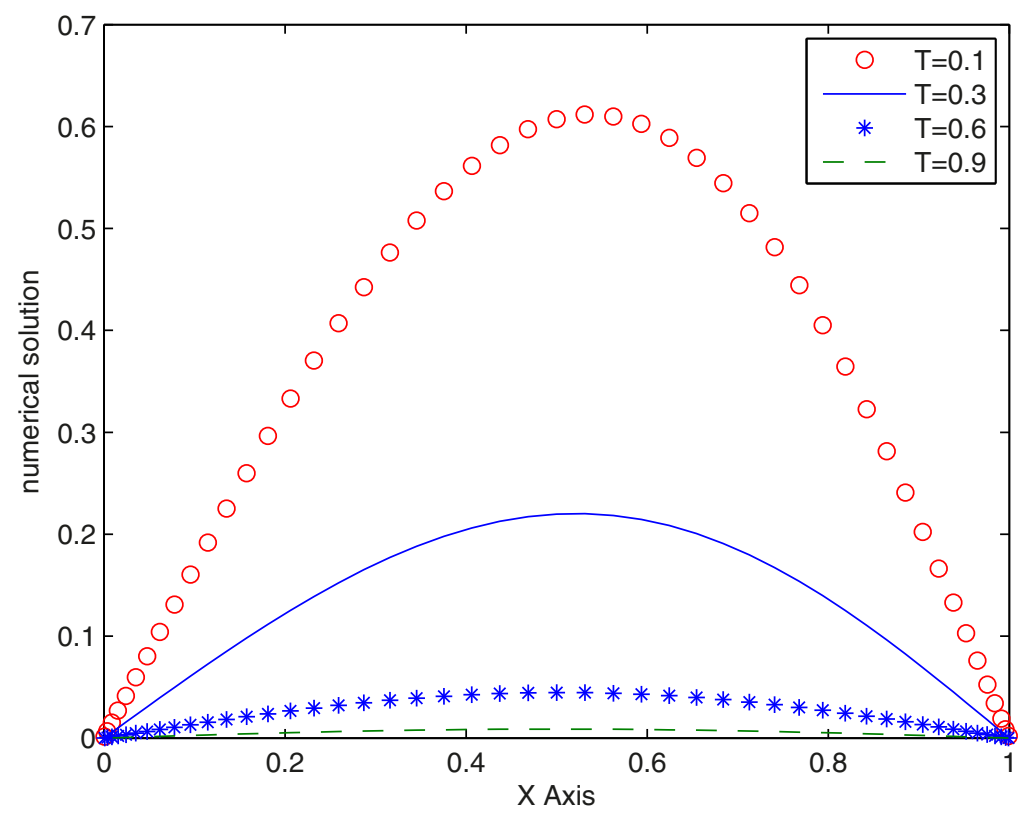

Fig. 1. Computed solutions of BHE for different values of time at $\epsilon=2^{-1}$.

Table 1

Convergence of Strang splitting for BHE at different values of $\epsilon$ and time.

\begin{tabular}{llllll}
\hline$T$ & $\epsilon$ & $N=8$ & $N=10$ & $N=20$ & $N=50$ \\
\hline 0.2 & $2^{-2}$ & 0.593005 & 0.593038 & 0.593037 & 0.593037 \\
& $2^{-16}$ & 0.870798 & 0.865167 & 0.865763 & 0.865763 \\
0.4 & $2^{-2}$ & 0.353251 & 0.353265 & 0.353265 & 0.353265 \\
& $2^{-16}$ & 0.502698 & 0.502690 & 0.502690 & 0.502690 \\
0.6 & $2^{-2}$ & 0.206562 & 0.206568 & 0.206568 & 0.206568 \\
& $2^{-16}$ & 0.023272 & 0.023861 & 0.023860 & 0.023860 \\
0.8 & $2^{-2}$ & 0.118291 & 0.118294 & 0.118294 & 0.118294 \\
& $2^{-16}$ & 0.030732 & 0.030741 & 0.030741 & 0.030741 \\
1.0 & $2^{-2}$ & 0.066753 & 0.066752 & 0.066752 & 0.066752 \\
& $2^{-16}$ & 0.029940 & 0.029945 & 0.029945 & 0.029945 \\
\hline
\end{tabular}

\section{Numerical results}

In this section, we numerically investigate the Strang splitting method. We consider the Burgers-Huxley equation in the form (2) for $\alpha=\beta=1, \gamma=0.5$, with initial and boundary conditions as follows [12]:

$$
\begin{aligned}
& u(x, 0)=\sin (\pi x), \quad 0 \leq x \leq 1 \\
& u(0, t)=u(1, t)=0, \quad 0 \leq t \leq T .
\end{aligned}
$$

When we apply the Strang splitting method on (2), we obtain the two subequations,

$$
\begin{aligned}
v_{t} & =A(v)=\epsilon v_{x x} \\
w_{t} & =B(w)=\beta(1-w)(w-\gamma) w-\alpha w w_{x}
\end{aligned}
$$

which are solved subsequently for small time steps $\Delta t$.

For the space discretization, we consider the Chebyshev differentiation matrices for the derivatives $u_{x}$ and $u_{x x}$. Semiimplicit Runge-Kutta method is used for the time integration, which is well-known for the numerical stability and less computational cost. The time step length $\Delta t=0.001$ is used for the numerical experiment.

The numerical results are presented in Figs. 1-3 for different values of $\epsilon$ and T. Fig. 4 shows the computed solution in $x-t$ plane.

The convergence of the Strang splitting solution is given by Table 1. It shows that if the grid point increases, we get the stable solution. Since there is no exact solution to BHE for given initial and boundary condition, we compare the numerical 


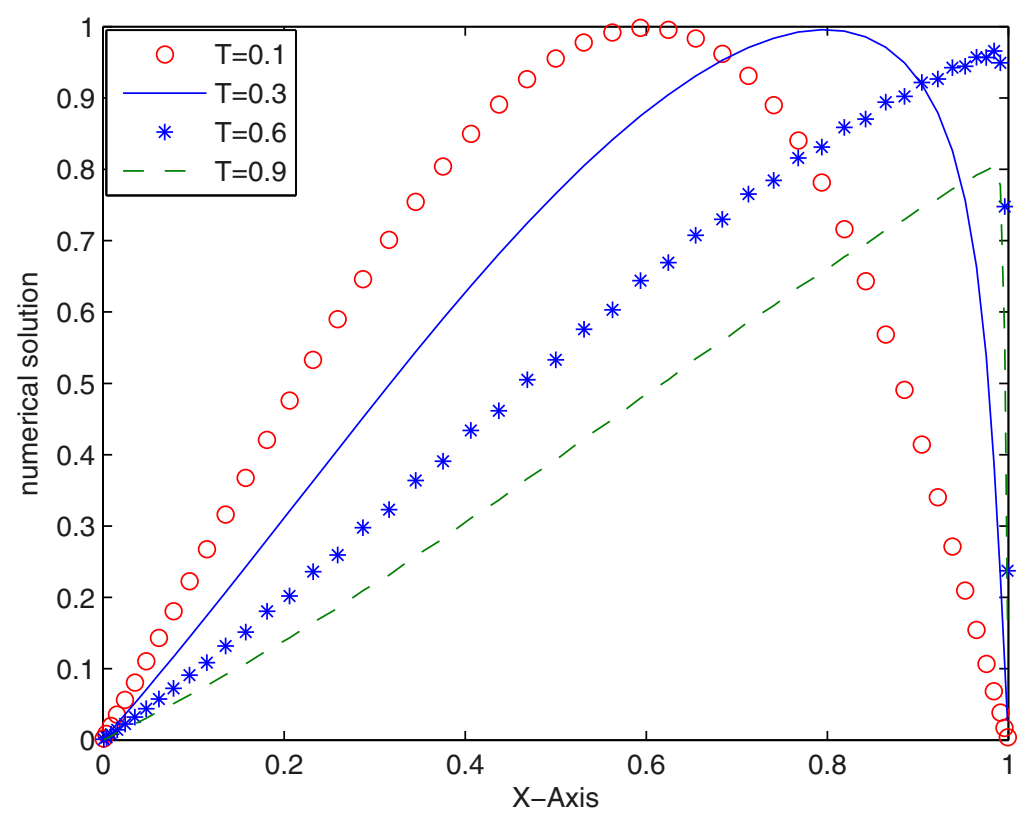

Fig. 2. Computed solutions of BHE for different values of time at $\epsilon=2^{-9}$.

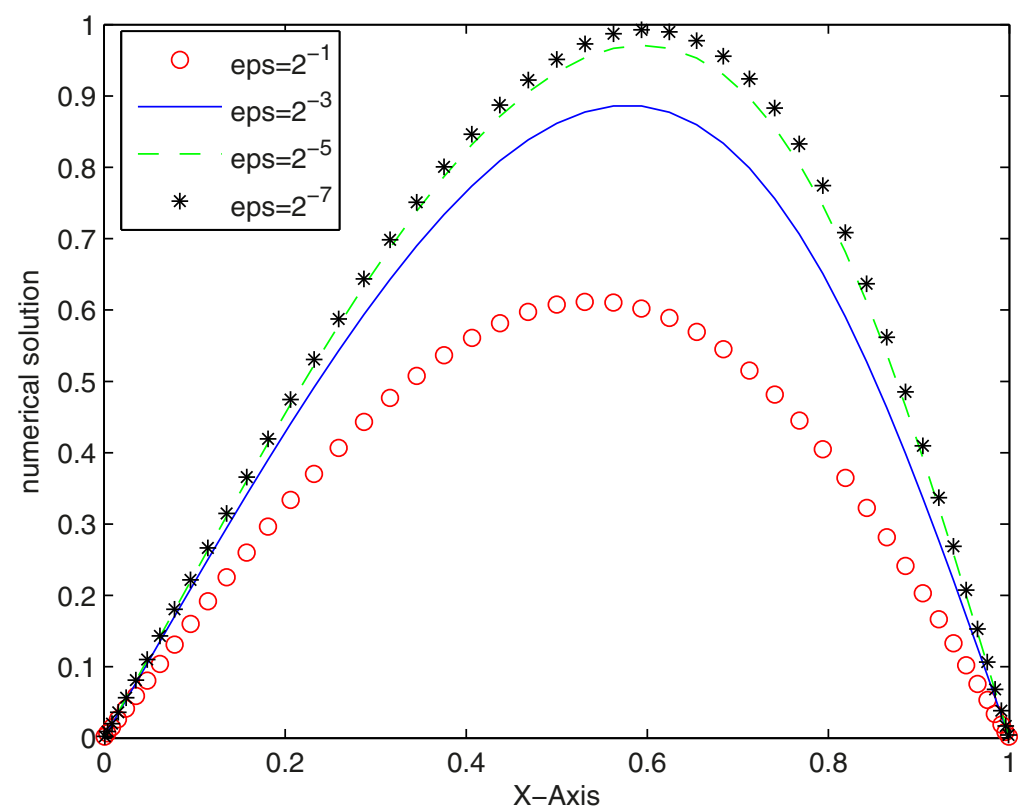

Fig. 3. Computed solutions of BHE for different values of $\epsilon$ at time $T=0.1$.

results with the reference solutions which are obtained by using the Differential Quadrature method [12]. These solutions for given time $T$ and space $x$ are given in Table 3 .

We solve the Burgers-Huxley equation in (2) by without splitting using the semi-implicit Runge-Kutta method and compare the Strang splitting solution with these solutions and get the following results.

The errors are given in Table 4 and Fig. 5, we see the expected orders for the Strang splitting method. InTable 2, numerical convergence rates are given for $\Delta t=0.001$ and $\Delta t=0.0005$. 
Table 2

Estimated errors and convergence rates for $\epsilon=2^{-9}$.

\begin{tabular}{llll}
\hline$T$ & $\epsilon=2^{-9}$ & & \\
\cline { 2 - 4 } & $\Delta t=0.001$ & $\Delta t=0.0005$ & Order \\
\hline 0.2 & 0.087082 & 0.022781 & 1.9345 \\
0.4 & 0.075154 & 0.020701 & 1.8601 \\
0.6 & 0.064051 & 0.018097 & 1.8234 \\
0.8 & 0.56221 & 0.015092 & 1.8976 \\
1 & 0.048867 & 0.010773 & 2.1818 \\
\hline
\end{tabular}

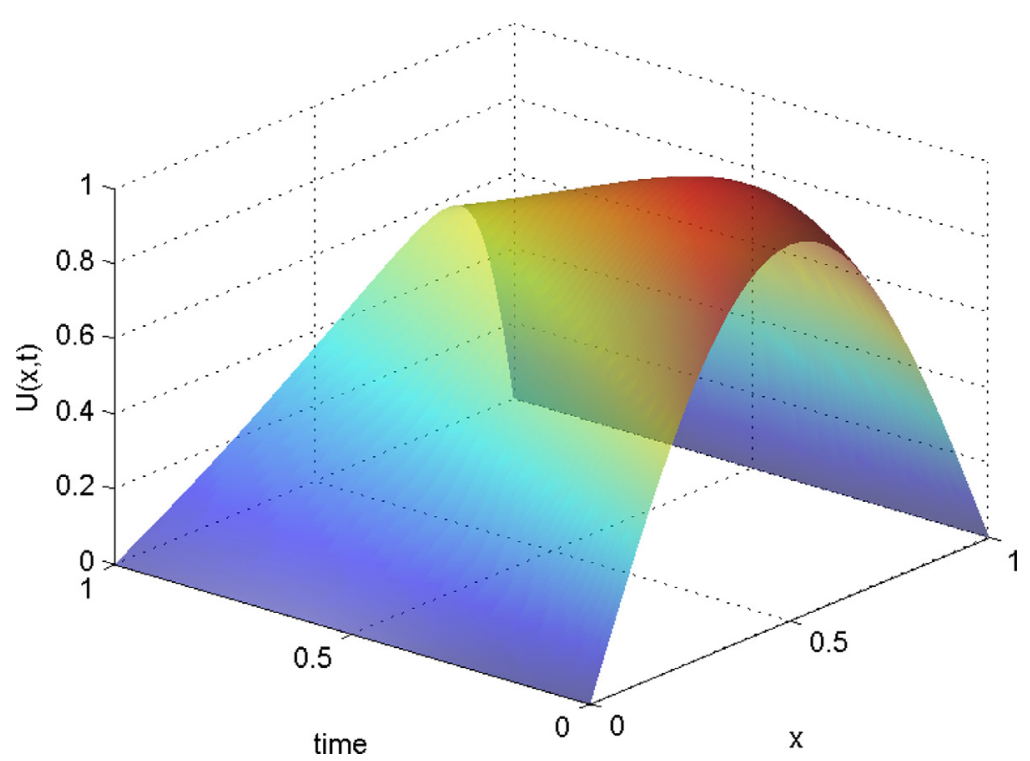

Fig. 4. Computed solutions of BHE for $\Delta t=0.001$ and $\epsilon=2^{-5}$.

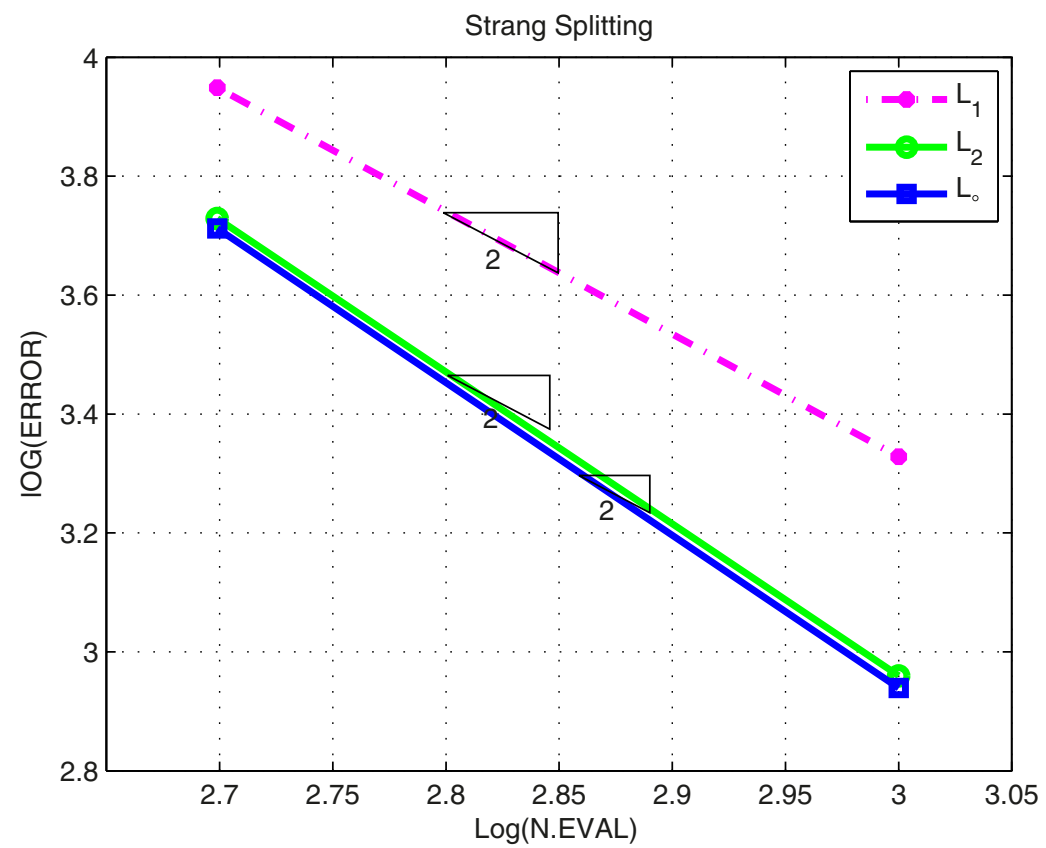

Fig. 5. Order of $L_{1}, L_{2}$ and $L_{\infty}$ errors. 
Table 3

Comparison between the split and the reference solution for $N=21$ and $\Delta t=0.001$.

\begin{tabular}{lllll}
\hline$T$ & $x$ & $\epsilon$ & Strang splitting solution & Reference solution \\
\hline 0.1 & 0.25 & $2^{-3}$ & 0.52586 & 0.52588 \\
& & $2^{-7}$ & 0.56849 & 0.56854 \\
0.9 & 0.25 & $2^{-3}$ & 0.15065 & 0.15062 \\
& & $2^{-7}$ & 0.18190 & 0.18172 \\
\hline
\end{tabular}

Table 4

Estimated errors for $\epsilon=2^{-9}$ at a fixed time $T$.

\begin{tabular}{llll}
\hline Time step & $L_{1}$ & $L_{2}$ & $L_{\infty}$ \\
\hline $10^{-3}$ & 0.0489 & 0.0103 & 0.0038 \\
$10^{-3} / 2$ & 0.0238 & 0.0050 & 0.0018 \\
$10^{-3} / 4$ & 0.0118 & 0.0025 & $9.0087 e-04$ \\
$10^{-3} / 8$ & 0.0059 & 0.0012 & $4.4465 e-04$ \\
$10^{-3} / 10$ & 0.0047 & $9.495 e-04$ & $3.5469 e-04$ \\
\hline
\end{tabular}

\section{Conclusion}

In this paper, we prove the second order convergence of the Strang splitting method in $H^{s}(\mathbb{R})$, for initial data in $H^{s+3}(\mathbb{R})$ for $s \geq 1$ for BHE. Comparing the proofs in this paper with those in [12], those in this text are easier. We considered the Strang splitting method for BHE and tested how well the method works. We divide the problem into linear and nonlinear parts and solve each subproblems connected the via-initial conditions.

In the numerical experimentation, since there is no exact solution of the BHE for given initial and boundary condition, we compare the numerical results with the reference solutions. We implement different solvers for the subequations from the splitting process, and test them to find a combination which works best for the Strang splitting. We observe that Chebyshev grid points produced accurate solutions. Comparing the technique in [12], we solve the BHE in a simpler way by using the Strang splitting.

\section{References}

[1] J. Satsuma, Topics in Soliton Theory and Exactly Solvable Nonlinear Equations, World Scientific, Singapore, 1987.

[2] H. Bateman, Some recent researches on the motion of fluids., Mon. Weather Rev. 43 (1915) 163-170.

[3] J.M. Burgers, Mathematical example illustrating relations occurring in the theory of turbulent fluid motion, Trans. R. Neth. Acad. Sci. Amsterdam 17 (1939) $1-53$.

[4] H. Holden, K.H. Karlsen, N.H. Risebro, T. Tao, Operator splitting for the KdC equation, Math. Comput. 80 (2011) 821-846.

[5] H. Holden, C. Lubich, N.H. Risebro, Operator splitting for partial differential equations with burger nonlinearity, Math. Comput. 82 (2013) 173-185.

[6] T. Jahnke, C. Lubich, Error bounds for exponential operator splitting, BIT 40 (2000) 735-744.

[7] C. Lubich, From Quantum to Classical Molecular Dynamics: Reduced Models and Numerical Analysis, European Mathematical Society, 2008.

[8] K.A. Bagrinovski, S.K. Godunov, Difference schemes for multidimensional problems, Dokl. Akad. Nauk SSSR (NS) 115 (1957) $413-433$.

[9] G. Strang, On the construction and comparison of different splitting schemes, SIAM J. Numer. Anal. 5 (3) (1968) $506-517$.

[10] G.I. Marchuk, Methods of Splitting, Nauka, Moscow, 1998.

[11] E.B. Nilsen, On operator splitting for the viscous Burgers' and the Korteweg-de Vries equations, M. Sc. Thesis (2011).

[12] R. Jiwari, R.C. Mittal, A higher order numerical scheme for singularly perturbed Burger-Huxley equation, J. Appl. Math. Inf. 29 (2011) 813-829,

[13] S. Zhou, X. Cheng, A linearly semi-implicit compact scheme for the Burger-Huxley equation, Int. J. Comput. Math. 88 (4) (2011) 795-804.

[14] J.E. Macas-Daz, J. Ruiz-Ramrez, J. Villa, The numerical solution of a generalized Burgers-Huxley equation through a conditionally bounded and symmetry-preserving method, Comput. Math. Appl. 61 (2011) 3330-3342.

[15] A.G. Bratsos, A fourth order improved numerical scheme for the generalized Burgers-Huxley equation, Am. J. Comput. Math. 1 (2011) 152-158.

[16] M. Sari, G. Grarslan, A. Zeytinoglu, High-order finite difference schemes for numerical solutions of the generalized Burgers-Huxley equation, Numer. Methods Partial Differ. Equ. 27 (2010) 1313-1326.

[17] R. Mohammadi, Hb-spline collocation algorithm for numerical solution of the generalized Burgers-Huxley equation, Numer. Methods Partial Differ. Equ. 29 (2012) 1173-1191. 